\title{
Knowledge on intrapartum care practices among skilled birth attendants in Cambodia-a cross-sectional study
}

\author{
Mitsuaki Matsui ${ }^{1 *}$ (1), Yuko Saito ${ }^{1}$, Rithy Po ${ }^{2}$, Bunsreng Taing ${ }^{3,4}$, Chamnan Nhek ${ }^{3,5}$, Rathavy Tung ${ }^{3,6}$, \\ Yoko Masaki ${ }^{3}$ and Azusa Iwamoto 3,7
}

\begin{abstract}
Background: Delivery is a critical moment for pregnant women and babies, and careful monitoring is essential throughout the delivery process. The partograph is a useful tool for monitoring and assessing labour progress as well as maternal and foetal conditions; however, it is often used inaccurately or inappropriately. A gap between practices and evidence-based guidelines has been reported in Cambodia, perhaps due to a lack of evidence-based knowledge in maternity care. This study aims to address to what extent skilled birth attendants in the first-line health services in Cambodia have knowledge on the management of normal delivery, and what factors are associated with their level of knowledge.
\end{abstract}

Methods: Midwives and nurses were recruited working in maternity in first-line public health facilities in Phnom Penh municipality, Kampong Cham and Svay Rieng provinces. Two self-administered questionnaires were applied. The first consisted of three sections with questions on monitoring aspects of the partograph: progress of labour, foetal, and maternal conditions. The second consisted of questions on diagnostic criteria, normal ranges, and standard intervals of monitoring during labour. A multiple linear regression analysis was performed to identify relationships between characteristics of the participants and the questionnaire scores.

Results: Of 542 eligible midwives and nurses, 523 (96\%) participated. The overall mean score was 58\%. Only 3\% got scores of more than $90 \%$. Multivariate analysis revealed that 'Kampong Cham province,','younger age', and 'higher qualification' were significantly associated with higher scores. Previous training experience was not associated with the score. Substantial proportions of misclassification of monitoring items during labour were found; for example, $61 \%$ answered uterine contraction as a foetal condition, and $44 \%$ answered foetal head descent and $26 \%$ answered foetal heart rate as a maternal condition.

Conclusion: This study found that knowledge was low on delivery management among skilled birth attendants. Previous training experience did not influence the knowledge level. A lack of understanding of physiology and anatomy was implied. Further experimental approaches should be attempted to improve the knowledge and quality of maternity services in Cambodia.

*Correspondence: mmatsui@nagasaki-u.ac.jp

${ }^{1}$ Department of Global Health, Nagasaki University School of Tropical Medicine and Global Health, Sakamoto 1-12-4, Nagasaki 852-8523, Japan

Full list of author information is available at the end of the article original author(s) and the source, provide a link to the Creative Commons licence, and indicate if changes were made. The images or other third party material in this article are included in the article's Creative Commons licence, unless indicated otherwise in a credit line to the material. If material is not included in the article's Creative Commons licence and your intended use is not permitted by statutory regulation or exceeds the permitted use, you will need to obtain permission directly from the copyright holder. To view a copy of this licence, visit http://creativecommons.org/licenses/by/4.0/. The Creative Commons Public Domain Dedication waiver (http://creativeco mmons.org/publicdomain/zero/1.0/) applies to the data made available in this article, unless otherwise stated in a credit line to the data. 


\begin{abstract}
Plain language summary
Pregnancy and childbirth are natural phenomena, but sometimes have risk for mothers and babies. Therefore, childbirth should be carefully and continuously monitored by the health care professional. The 'partograph' is a useful tool that defines three monitoring aspects of the delivery progress, and conditions of the mother and intrauterine baby. However, it is often used inaccurately or inappropriately in low- and middle-income countries. We hypothesised that health professionals who assist childbirth cannot effectively monitor delivery conditions because their knowledge is insufficient. Therefore, we evaluated the knowledge on monitoring the process of childbirth and explored factors which affect the level of knowledge among health care providers in Cambodia.

Midwives and nurses were targeted in this study who deal with normal deliveries in the capital city and two provinces. The questionnaire was designed to evaluate if their knowledge on three monitoring aspects is accurate.

Of 542 eligible personnel, 523 (96\%) participated. The mean score was 58\%. Only 3\% got scores of more than 90\%. According to the statistical analysis, 'working in Kampong Cham province', 'younger age', and 'higher qualification' were significantly associated with higher scores. Previous training experience was not associated with the score.

This study found that basic knowledge was low on delivery management among health care providers. We suspect that a deficiency of basic medical knowledge, such as physiology and anatomy, causes the lack of knowledge on the childbirth process. Further intervention should be attempted to improve the knowledge and quality of maternity services in Cambodia.
\end{abstract}

Keywords: Skilled birth attendant, Midwifery (MeSH terms), Intrapartum care, Cambodia (MeSH terms), Partograph

\section{Introduction}

The process of delivery and childbirth is characterised by dynamic changes in anatomy and physiology for mother and foetus. As delivery progresses, uterine contractions become intense and the foetus in utero is exposed to extremely low levels of oxygen [1]. Asphyxia is the most common cause of neonatal deaths in south Asia and subSaharan Africa [2]. Delivery is also a critical moment for pregnant women, and it has been estimated that $28 \%$ of maternal deaths in the world occur during the intrapartum or immediate postpartum period [3]. Another estimate is that $40-45 \%$ of maternal deaths, stillbirths, and neonatal deaths occur during or within $24 \mathrm{~h}$ after delivery in south Asia and sub-Saharan Africa [2]. Therefore, to ensure a normal birth process careful monitoring is essential throughout the delivery procedure so that abnormal signs are detected early.

The partograph is a useful tool for monitoring and assessing labour progress as well as maternal and foetal conditions [4]. It defines the key items to be monitored, their normal ranges, and the standard monitoring intervals. Although the latest recommendations from WHO emphasise to not follow the cervical dilatation rate threshold of $1 \mathrm{~cm}$ per hour during the active phase in the first stage of labour [5], the usefulness of the partograph is not hampered. It is still necessary to plot cervical dilatation versus time on the cervicograph to confirm the progress of labour. Therefore, the utilisation of the partograph helps to identify labour dystocia and risks for adverse birth outcomes [6]. However, it has been shown that the partograph is often used inaccurately or inappropriately, particularly in low- and middle-income countries [7-11].

Cambodia successfully decreased maternal mortality to the target set in the Millennium Development Goals (MDGs) [12]. The latest Cambodia Demographic and Health Survey in 2014 has shown that the maternal mortality ratio and the proportion of deliveries attended by skilled health professionals were 170 per 100,000 live births and 89\%, respectively [13]. The Cambodian Ministry of Health has built on the MDGs success and has placed maternal mortality reduction at the top of its third Strategic Health Plan 2016-2020 [14]. Improving the quality of health services is a key strategy for reduction and evidence-based practice is a major element of quality of care. This initiative corresponds with the desire to increase the competency of skilled birth attendants (SBA), which was recently redefined jointly by WHO, UNFPA, UNICEF, ICM, ICN, FIGO, and IPA. It includes: (i) to provide and promote evidence-based, human-rights-based, quality, socio-culturally sensitive, and dignified care to women and newborns; (ii) to facilitate physiological processes during labour and delivery to ensure a clean and positive childbirth experience; and (iii) to identify and manage or refer women and/or newborns with complications [15]. Competency is measured by performance, which is based on the knowledge, skills, and behaviours required as a professional.

Studies in Cambodia revealed that there was a gap between practices and evidence-based guidelines, although substantial technical support in obstetric and midwifery training courses had been provided for two 
decades [16-20]. One possible reason behind this gap is a lack of evidence-based knowledge in maternity care [16, $20]$. However, there has been no systematic assessment of knowledge on intrapartum care practices among skilled birth attendants in Cambodia.

\section{Methods}

\section{Study aims}

This study aimed to address two questions: (i) to what extent skilled birth attendants in the first-line health services in Cambodia have sufficient knowledge on management of intrapartum care; and (ii) what factors are associated with the level of knowledge, especially previous training experiences among the health care providers.

\section{Study design}

This study employed a cross-sectional design.

\section{Study setting}

This study was jointly organised by a research project on the impact of 'evidence-based midwifery care' on maternal and neonatal outcomes, and a bilateral technical cooperation project between the Cambodian and Japanese governments. The former was conducted in selected first-line public health facilities in the capital city Phnom Penh. The latter is implemented by the National Maternal and Child Health Centre (NMCHC) in Cambodia and Japan International Cooperation Agency (JICA), called 'Project for Improving Continuum of Care with focus on Intrapartum and Neonatal Care in Cambodia' (IINeoC). Its target areas are Kampong Cham and Svay Rieng provinces, which are in the central lowlands of the Mekong river and in southeastern Cambodia next to Vietnam, respectively. This study was conducted as a baseline survey in the two projects.

\section{Target facility and population}

Midwives and nurses were recruited working in maternity in the first-line public health facilities in Kampong Cham and Svay Rieng provinces, and Phnom Penh municipality. Health facilities which had 20 deliveries or more per month were selected for Phnom Penh, whereas all facilities were involved in Kampong Cham and Svay Rieng provinces. A list of health care personnel assisting deliveries was prepared prior to the survey. All participants were invited to the municipal or provincial health departments. The questionnaires were applied to those who agreed to participate. Data collection was conducted between 11 and 15 June 2018 in Svay Rieng, 9 and 12 July 2018 in Kampong Cham, and 16 and 17 July 2018 in Phnom Penh.

\section{Measurement of knowledge}

Two sets of self-administered questionnaires were prepared to measure knowledge on intrapartum care. The first questionnaire consisted of three sections with questions on monitoring items for three aspects of the partograph: progress of labour, foetal condition, and maternal condition. Every question was open-ended; therefore, the participants were asked to conduct free listing of the items. The answers were scored, and one point was given for each correct item specified by a participant. There are ten correct items in the first questionnaire, and therefore the total score ranges between zero and ten. After completion of the first questionnaire, the second one was distributed. It has sixteen questions which consist of nine items on knowledge of criteria of diagnosis or the normal range of diagnostic parameters, and seven questions on standard interval of monitoring during labour. Each question in the second questionnaire had a single correct answer except for questions on diagnosis of hypertension. The questions on hypertension displayed 42 different results of blood pressure and the participant was asked to distinguish instances of hypertension. A score of 0.024 was given for each of 42 correct answers. One point was given to each correct answer in the other questions. All twenty-six components of the questionnaire with the expected answers are shown in Table 1.

The most recent national protocol was referred, Safe Motherhood Clinical Management Protocols for Health Centers (2016) [21]. A draft of the questionnaire was prepared by the researchers (MM, YS, YM, and $\mathrm{AI})$, and to make a final version it was consulted in a meeting involving obstetrics and maternity care experts from the Cambodian Society of Gynaecology and Obstetrics, the Cambodian Midwives Association, and NMCHC. The questionnaires were made in the Cambodian language (Khmer), which was confirmed by backtranslation into English. All technical terms were taken from the protocol.

\section{Information on study participants}

Information was collected on the participants regarding age, qualification, number of deliveries assisted in the prior month, and previous training experience. We selected three training courses as potential contributing factors on knowledge improvement, because these include a topic of monitoring and evaluation during labour. The titles of the courses are 'Health centre midwifery course in NMCHC (HC-MW)', 'Partograph', and 'Basic Emergency Obstetric and Neonatal Care (BEmONC).' The details of the training are described in Annex 1 (as an Additional file 1). 
Table 1 Contents of the questionnaires to measure knowledge on intrapartum care

\begin{tabular}{|c|c|c|}
\hline \multicolumn{3}{|c|}{ I. Knowledge on monitoring items ( 10 items) } \\
\hline \multicolumn{2}{|l|}{ Category } & Expected answer \\
\hline \multirow{3}{*}{\multicolumn{2}{|c|}{ Progress of labour }} & Cervical dilatation \\
\hline & & Uterine contraction \\
\hline & & Foetal head descent \\
\hline \multirow{3}{*}{\multicolumn{2}{|c|}{ Foetal condition }} & Foetal heart rate (FHR) \\
\hline & & Amniotic fluid \\
\hline & & Moulding of foetal head \\
\hline \multirow{4}{*}{\multicolumn{2}{|c|}{ Maternal condition }} & Pulse \\
\hline & & Blood pressure \\
\hline & & Body temperature \\
\hline & & Urine volume \\
\hline \multicolumn{3}{|c|}{ II-1. Knowledge on criteria for diagnosis or normal range of the items (9 items) } \\
\hline Category & Question & Expected answer \\
\hline \multirow[t]{5}{*}{ Progress of labour } & Beginning of active phase in the 1st stage & From $3 \mathrm{~cm}$ of dilatation of uterine cervix \\
\hline & End of 1st stage; or beginning of 2nd stage & At full dilatation of uterine cervix \\
\hline & End of 2 nd stage; or beginning of 3rd stage & At expulsion of baby \\
\hline & End 3rd stage; or beginning of 4th stage & At expulsion of placenta \\
\hline & End of 4 th stage & $2 \mathrm{~h}$ after delivery \\
\hline \multirow[t]{2}{*}{ Foetal condition } & Upper normal limit of FHR & 160 beats per minutes \\
\hline & Lower normal limit of FHR & 120 beats per minutes \\
\hline \multirow[t]{2}{*}{ Maternal condition } & Diagnosis of hypertension & $\begin{array}{l}\text { Systolic blood pressure } 140 \mathrm{~mm} \mathrm{Hg} \text { or more; or Diastolic } \\
\text { blood pressure } 90 \mathrm{~mm} \mathrm{Hg} \text { or more }\end{array}$ \\
\hline & Abnormal amount of PPH & $500 \mathrm{ml}$ or more \\
\hline \multicolumn{3}{|c|}{ II-2. Knowledge on recommended interval of monitoring ( 7 items) } \\
\hline Category & Question & Expected answer \\
\hline \multirow[t]{2}{*}{ Progress of labour } & Cervical dilatation, 1st stage & Every $4 \mathrm{~h}$ \\
\hline & Uterine contraction, 1st stage & Every 30 min \\
\hline \multirow[t]{2}{*}{ Foetal condition } & FHR, 1st stage & Every 30 min \\
\hline & FHR, 2nd stage & Every 5 min \\
\hline \multirow[t]{2}{*}{ Maternal condition } & Pulse & Every $1 \mathrm{~h}$ \\
\hline & Blood pressure & Every $1 \mathrm{~h}$ \\
\hline
\end{tabular}

\section{Data processing and analysis}

The sum points for each participant were calculated. Univariate analyses by Student's t-test or analysis of variance, and a multiple linear regression analysis were performed to identify the relationships between the characteristics of the participants and their received score, using Stata version 15 (Stata Corp, USA).

\section{Results}

\section{Participants and their characteristics}

Of 542 eligible health care personnel in the three regions, 523 (96\%) from 157 health facilities participated. Characteristics of the participants are shown in Table 2 .
Distribution of age was bimodal with modes in the twenties and forties. Midwife was dominant as a qualification. The median number of deliveries assisted was higher in Phnom Penh than in the two provinces. Previous experiences of attending obstetrics and midwifery training courses differed by region.

\section{Knowledge on intrapartum care}

Table 3a shows that the overall mean score was $58 \%$ (SD 19\%). Only 3\% of the participants scored more than $90 \%$. Table 3b shows knowledge on monitoring items using the partograph. The lowest correct answer rates were for moulding (18\%), amniotic fluid (44\%), maternal 
Table 2 Characteristics of the participants, by province

\begin{tabular}{|c|c|c|c|c|c|c|c|c|}
\hline Province & Phnom Penh & & Kampong Cham & & Svay Rieng & & Total & \\
\hline Number of participants & 101 & & 255 & & 167 & & 523 & \\
\hline Number of health facilities & 17 & & 93 & & 47 & & 157 & \\
\hline \multicolumn{9}{|l|}{ Age } \\
\hline $21-25$ & 14 & $14 \%$ & 29 & $11 \%$ & 34 & $20 \%$ & 77 & $15 \%$ \\
\hline $26-30$ & 35 & $35 \%$ & 97 & $38 \%$ & 79 & $47 \%$ & 211 & $40 \%$ \\
\hline $31-35$ & 15 & $15 \%$ & 32 & $13 \%$ & 19 & $11 \%$ & 66 & $13 \%$ \\
\hline $36-40$ & 4 & $4 \%$ & 7 & $3 \%$ & 2 & $1 \%$ & 13 & $2 \%$ \\
\hline $41-45$ & 7 & $7 \%$ & 20 & $8 \%$ & 2 & $1 \%$ & 29 & $6 \%$ \\
\hline $46-50$ & 18 & $18 \%$ & 47 & $18 \%$ & 14 & $8 \%$ & 79 & $15 \%$ \\
\hline $51-55$ & 2 & $2 \%$ & 12 & $5 \%$ & 11 & $7 \%$ & 25 & $5 \%$ \\
\hline $56-60$ & 6 & $6 \%$ & 11 & $4 \%$ & 6 & $4 \%$ & 23 & $4 \%$ \\
\hline Median (IQR) & $31(27-46)$ & & $31(28-46)$ & & $28(26-33)$ & & $30(27-45)$ & \\
\hline \multicolumn{9}{|l|}{ Qualification and degree } \\
\hline \multicolumn{9}{|l|}{ Midwife } \\
\hline Bachelor & 11 & $11 \%$ & 18 & $7 \%$ & 6 & $4 \%$ & 35 & $7 \%$ \\
\hline Secondary & 69 & $68 \%$ & 143 & $56 \%$ & 99 & $59 \%$ & 311 & $59 \%$ \\
\hline Primary & 8 & $8 \%$ & 69 & $27 \%$ & 55 & $33 \%$ & 132 & $25 \%$ \\
\hline \multicolumn{9}{|l|}{ Nurse } \\
\hline Bachelor & 8 & $8 \%$ & 20 & $8 \%$ & 3 & $2 \%$ & 31 & $6 \%$ \\
\hline Secondary & 4 & $4 \%$ & 2 & $1 \%$ & 1 & $1 \%$ & 7 & $1 \%$ \\
\hline Primary & 1 & $1 \%$ & 3 & $1 \%$ & 3 & $2 \%$ & 7 & $1 \%$ \\
\hline \multicolumn{9}{|c|}{ Number of deliveries assisted in previous month } \\
\hline 2 or less & 19 & $19 \%$ & 99 & $39 \%$ & 64 & $38 \%$ & 182 & $35 \%$ \\
\hline $3-5$ & 26 & $26 \%$ & 88 & $35 \%$ & 65 & $39 \%$ & 179 & $34 \%$ \\
\hline $6-10$ & 32 & $32 \%$ & 61 & $24 \%$ & 32 & $19 \%$ & 125 & $24 \%$ \\
\hline 11 or more & 24 & $24 \%$ & 7 & $3 \%$ & 6 & $4 \%$ & 37 & $7 \%$ \\
\hline Median (IQR) & $6(3-10)$ & & $3(2-6)$ & & $3(2-5)$ & & $4(2-7)$ & \\
\hline \multicolumn{9}{|l|}{ Previous training experience } \\
\hline Health Centre Midwife & 11 & $11 \%$ & 60 & $24 \%$ & 32 & $19 \%$ & 103 & $20 \%$ \\
\hline Partograph & 33 & $33 \%$ & 107 & $42 \%$ & 55 & $33 \%$ & 195 & $37 \%$ \\
\hline Basic EmONC & 50 & $50 \%$ & 46 & $18 \%$ & 87 & $52 \%$ & 183 & $35 \%$ \\
\hline
\end{tabular}

body temperature (41\%), and maternal pulse (44\%). For knowledge on criteria for diagnosis, only $62 \%$ and $49 \%$ of the participants correctly answered the questions on 'commencement of active phase of the first stage of labour' and 'diagnosis criteria for post-partum haemorrhage', respectively. For the knowledge on recommended intervals of monitoring, the correct answer rates were between 33 and 57\%. This indicates that more than half of the participants do not know the standard methods of monitoring during labour.

\section{Correlating factors with knowledge on process of delivery}

Tables 4 and 5 show the results of univariate and multivariate analyses between the characteristics of the participants and their score on the knowledge test, respectively. Univariate analyses revealed that province, age, qualification, and several previous training experiences had statistically significant associations with the score. However, those who had attended health centre midwife and partograph training courses significantly scored less than those who have not received the trainings. The number of deliveries assisted was not associated with the score.

Multivariate linear regression analysis revealed that 'Kampong Cham province', 'younger age', and 'higher qualification' were significantly associated with a higher score. Previous experience in training courses was not associated with the score.

\section{Misclassification of monitoring items during delivery}

The proportion of wrong answers in the three questions on items to be monitored during labour is shown in Table 6. Sixty-one percent of the participants answered 'uterine contraction' as an item of foetal condition. 'Cervical dilatation' and 'foetal head descent' were 
Table 3 Knowledge on intrapartum care

\begin{tabular}{|c|c|c|c|c|c|c|c|c|}
\hline \multicolumn{9}{|c|}{ a. Overall score in $\mathbf{2 6}$ items } \\
\hline \multirow{2}{*}{$\begin{array}{l}\text { Province } \\
\text { [n] }\end{array}$} & \multicolumn{2}{|l|}{ Phnom Penh } & \multicolumn{2}{|l|}{ Kampong Cham } & \multicolumn{2}{|l|}{ Svay Rieng } & \multicolumn{2}{|l|}{ Total } \\
\hline & [101] & & {$[255]$} & & [167] & & [523] & \\
\hline $0-10 \%$ & 1 & $1 \%$ & 0 & $0 \%$ & 0 & $0 \%$ & 1 & $0 \%$ \\
\hline $11-20 \%$ & 4 & $4 \%$ & 1 & $0 \%$ & 10 & $6 \%$ & 15 & $3 \%$ \\
\hline $21-30 \%$ & 7 & $7 \%$ & 7 & $3 \%$ & 12 & $7 \%$ & 26 & $5 \%$ \\
\hline $31-40 \%$ & 13 & $13 \%$ & 19 & $7 \%$ & 26 & $16 \%$ & 58 & $11 \%$ \\
\hline $41-50 \%$ & 17 & $17 \%$ & 33 & $13 \%$ & 26 & $16 \%$ & 76 & $15 \%$ \\
\hline $51-60 \%$ & 17 & $17 \%$ & 37 & $15 \%$ & 30 & $18 \%$ & 84 & $16 \%$ \\
\hline $61-70 \%$ & 18 & $18 \%$ & 58 & $23 \%$ & 36 & $22 \%$ & 112 & $21 \%$ \\
\hline $71-80 \%$ & 16 & $16 \%$ & 59 & $23 \%$ & 19 & $11 \%$ & 94 & $18 \%$ \\
\hline $81-90 \%$ & 5 & $5 \%$ & 30 & $12 \%$ & 7 & $4 \%$ & 42 & $8 \%$ \\
\hline $91-100 \%$ & 3 & $3 \%$ & 11 & $4 \%$ & 1 & $1 \%$ & 15 & $3 \%$ \\
\hline Mean (SD) & $54.1 \%(19.3 \%)$ & & $63.2 \%(17.1 \%)$ & & $51.5 \%(18.5 \%)$ & & $57.7 \%$ (18.7\%) & \\
\hline
\end{tabular}

\section{b. Scores by each item}

\begin{tabular}{|c|c|c|c|c|c|c|c|c|c|}
\hline \multicolumn{2}{|l|}{ Province } & \multirow{2}{*}{\multicolumn{2}{|c|}{$\begin{array}{l}\text { Phnom Penh } \\
{[101]}\end{array}$}} & \multirow{2}{*}{\multicolumn{2}{|c|}{$\frac{\text { Kampong Cham }}{[255]}$}} & \multirow{2}{*}{\multicolumn{2}{|c|}{$\frac{\text { Svay Rieng }}{[167]}$}} & \multirow{2}{*}{\multicolumn{2}{|c|}{$\frac{\text { Total }}{[523]}$}} \\
\hline & {$[n]$} & & & & & & & & \\
\hline \multicolumn{10}{|c|}{ I. Knowledge on monitoring items } \\
\hline \multirow[t]{3}{*}{ Progress of labour } & Cervical dilatation & 69 & $68 \%$ & 196 & $77 \%$ & 106 & $63 \%$ & 371 & $71 \%$ \\
\hline & Uterine contraction & 74 & $73 \%$ & 207 & $81 \%$ & 130 & $78 \%$ & 411 & $79 \%$ \\
\hline & Foetal head descent & 58 & $57 \%$ & 154 & $60 \%$ & 77 & $46 \%$ & 289 & $55 \%$ \\
\hline \multirow[t]{3}{*}{ Foetal conditions } & Foetal heart rate & 97 & $96 \%$ & 250 & $98 \%$ & 149 & $89 \%$ & 496 & $95 \%$ \\
\hline & Amniotic fluid & 50 & $50 \%$ & 130 & $51 \%$ & 49 & $29 \%$ & 229 & $44 \%$ \\
\hline & Moulding of foetal head & 20 & $20 \%$ & 68 & $27 \%$ & 4 & $2 \%$ & 92 & $18 \%$ \\
\hline \multirow[t]{4}{*}{ Maternal conditions } & Pulse & 50 & $50 \%$ & 121 & $47 \%$ & 57 & $34 \%$ & 228 & $44 \%$ \\
\hline & Blood pressure & 69 & $68 \%$ & 148 & $58 \%$ & 89 & $53 \%$ & 306 & $59 \%$ \\
\hline & Body temperature & 41 & $41 \%$ & 114 & $45 \%$ & 57 & $34 \%$ & 212 & $41 \%$ \\
\hline & Urine volume & 42 & $42 \%$ & 173 & $68 \%$ & 62 & $37 \%$ & 277 & $53 \%$ \\
\hline \multicolumn{10}{|c|}{ II. Knowledge on criteria for diagnosis or normal range of the items } \\
\hline \multirow[t]{5}{*}{ Progress of labour } & Commencement of active phase & 63 & $62 \%$ & 150 & $59 \%$ & 110 & $66 \%$ & 323 & $62 \%$ \\
\hline & End of the 1st stage & 71 & $70 \%$ & 234 & $92 \%$ & 116 & $69 \%$ & 421 & $80 \%$ \\
\hline & End of the 2nd stage & 77 & $76 \%$ & 230 & $90 \%$ & 119 & $71 \%$ & 426 & $81 \%$ \\
\hline & End of the 3rd stage & 71 & $70 \%$ & 199 & $78 \%$ & 116 & $69 \%$ & 386 & $74 \%$ \\
\hline & End of the 4th stage & 48 & $48 \%$ & 164 & $64 \%$ & 87 & $52 \%$ & 299 & $57 \%$ \\
\hline \multirow[t]{2}{*}{ Foetal conditions } & Upper normal limit of FHR & 72 & $71 \%$ & 197 & $77 \%$ & 129 & $77 \%$ & 398 & $76 \%$ \\
\hline & Lower normal limit of FHR & 67 & $66 \%$ & 193 & $76 \%$ & 119 & $71 \%$ & 379 & $72 \%$ \\
\hline \multirow[t]{8}{*}{ Maternal conditions } & Diagnosis of hypertension (correct & answer rate) & & & & & & & \\
\hline & $0-20 \%$ & 5 & $5 \%$ & 12 & $5 \%$ & 10 & $6 \%$ & 27 & $5 \%$ \\
\hline & $21-40 \%$ & 4 & $4 \%$ & 9 & $4 \%$ & 8 & $5 \%$ & 21 & $4 \%$ \\
\hline & $41-60 \%$ & 15 & $15 \%$ & 41 & $16 \%$ & 18 & $11 \%$ & 74 & $14 \%$ \\
\hline & $61-80 \%$ & 27 & $27 \%$ & 108 & $42 \%$ & 71 & $43 \%$ & 206 & $39 \%$ \\
\hline & $81-100 \%$ & 50 & $50 \%$ & 85 & $33 \%$ & 60 & $36 \%$ & 195 & $37 \%$ \\
\hline & Median (IQR) & $79 \%$ & $(62-86 \%)$ & $71 \%$ & $(62-83 \%)$ & $73 \%$ & $(62-83 \%)$ & $74 \%$ & $(62-83 \%)$ \\
\hline & Diagnosis of PPH & 44 & $44 \%$ & 149 & $58 \%$ & 62 & $37 \%$ & 255 & $49 \%$ \\
\hline \multicolumn{10}{|c|}{ III. Knowledge on recommended interval of monitoring } \\
\hline \multirow[t]{2}{*}{ Progress of labour } & Cervical dilataion, 1st stage & 43 & $43 \%$ & 137 & $54 \%$ & 90 & $54 \%$ & 270 & $52 \%$ \\
\hline & Uterine contraction, 1st stage & 26 & $26 \%$ & 87 & $34 \%$ & 57 & $34 \%$ & 170 & $33 \%$ \\
\hline
\end{tabular}


Table 3 (continued)

\begin{tabular}{|c|c|c|c|c|c|c|c|c|c|}
\hline \multicolumn{10}{|l|}{ b. Scores by each item } \\
\hline \multicolumn{2}{|l|}{ Province } & \multirow{2}{*}{$\frac{\text { Phnom Penh }}{\text { [101] }}$} & & \multicolumn{2}{|l|}{ Kampong Cham } & \multicolumn{2}{|l|}{ Svay Rieng } & \multicolumn{2}{|l|}{ Total } \\
\hline & {$[\mathrm{n}]$} & & & {$[255]$} & & [167] & & [523] & \\
\hline \multirow[t]{2}{*}{ Foetal conditions } & FHR, 1st stage & 48 & $48 \%$ & 169 & $66 \%$ & 79 & $47 \%$ & 296 & $57 \%$ \\
\hline & FHR, 2nd stage & 23 & $23 \%$ & 132 & $52 \%$ & 39 & $23 \%$ & 194 & $37 \%$ \\
\hline \multirow[t]{3}{*}{ Maternal conditions } & Pulse rate & 35 & $35 \%$ & 106 & $42 \%$ & 55 & $33 \%$ & 196 & $37 \%$ \\
\hline & Blood pressure & 43 & $43 \%$ & 113 & $44 \%$ & 51 & $31 \%$ & 207 & $40 \%$ \\
\hline & Body temperature & 33 & $33 \%$ & 115 & $45 \%$ & 53 & $32 \%$ & 201 & $38 \%$ \\
\hline
\end{tabular}

Table 4 Relationship between characteristics of the participants and level of knowledge, univariate analysis

\begin{tabular}{|c|c|c|c|c|c|}
\hline \multicolumn{2}{|l|}{ Characteristics } & $\mathrm{n}$ & Score (mean) & [SD] & $p$-value \\
\hline \multicolumn{6}{|l|}{ Province } \\
\hline \multicolumn{2}{|l|}{ Phnom Penh } & 101 & $54.1 \%$ & $19.3 \%$ & $<0.001$ \\
\hline \multicolumn{2}{|l|}{ Kampong Cham } & 255 & $63.2 \%$ & $17.1 \%$ & \\
\hline \multicolumn{2}{|l|}{ Svay Rieng } & 167 & $51.5 \%$ & $18.5 \%$ & \\
\hline \multicolumn{6}{|l|}{ Age } \\
\hline \multicolumn{2}{|l|}{$21-30$} & 288 & $62.4 \%$ & $17.0 \%$ & $<0.001$ \\
\hline \multicolumn{2}{|l|}{$31-40$} & 79 & $59.8 \%$ & $18.4 \%$ & \\
\hline \multicolumn{2}{|l|}{$41-50$} & 108 & $49.3 \%$ & $17.8 \%$ & \\
\hline \multicolumn{2}{|l|}{$51-60$} & 48 & $45.2 \%$ & $19.7 \%$ & \\
\hline \multicolumn{6}{|l|}{ Qualification } \\
\hline \multirow[t]{3}{*}{ Midwife } & Bachelor & 35 & $65.6 \%$ & $16.0 \%$ & $<0.001$ \\
\hline & Secondary & 309 & $60.2 \%$ & $17.7 \%$ & \\
\hline & Primary & 132 & $48.7 \%$ & $17.2 \%$ & \\
\hline \multirow[t]{3}{*}{ Nurse } & Bachelor & 33 & $70.0 \%$ & $17.2 \%$ & \\
\hline & Secondary & 7 & $44.0 \%$ & $25.3 \%$ & \\
\hline & Primary & 7 & $35.2 \%$ & $17.5 \%$ & \\
\hline \multicolumn{6}{|c|}{ Number of deliveries assisted (per month) } \\
\hline \multicolumn{2}{|c|}{2 or less } & 182 & $56.4 \%$ & $19.7 \%$ & 0.16 \\
\hline \multicolumn{2}{|l|}{$3-5$} & 179 & $59.9 \%$ & $17.0 \%$ & \\
\hline \multicolumn{2}{|l|}{6 or more } & 162 & $56.8 \%$ & $19.4 \%$ & \\
\hline \multicolumn{6}{|c|}{ Previous training experience } \\
\hline \multirow{2}{*}{$\begin{array}{l}\text { Health center } \\
\text { midwife }\end{array}$} & Yes & 103 & $53.4 \%$ & $19.2 \%$ & 0.009 \\
\hline & No & 420 & $58.8 \%$ & $18.5 \%$ & \\
\hline \multirow[t]{2}{*}{ Partograph } & Yes & 195 & $54.0 \%$ & $17.4 \%$ & $<0.001$ \\
\hline & No & 328 & $59.9 \%$ & $19.2 \%$ & \\
\hline \multirow[t]{2}{*}{ EmONC } & Yes & 183 & $58.2 \%$ & $20.0 \%$ & 0.68 \\
\hline & No & 340 & $57.5 \%$ & $18.1 \%$ & \\
\hline
\end{tabular}

categorised as a maternal condition by 42 and $44 \%$ of the participants, respectively. 'Amniotic fluid' was categorised as progress of labour by $35 \%$. Vital signs of a mother were categorised as progress of labour by between 20 and $29 \%$.
Table 5 Relationship between characteristics of the participants and level of knowledge, multiple linear regression analysis

\begin{tabular}{lclc}
\hline Factors & B-coeffient & $\mathbf{( 9 5 \% ~ C l )}$ & $\boldsymbol{p}$-value \\
\hline $\begin{array}{l}\text { Province } \\
\text { Phnom Penh }\end{array}$ & 2.4 & $(-1.9,6.6)$ & 0.27 \\
Kampong Cham & 12.7 & $(9.4,16.0)$ & $<0.001$ \\
$\quad$ Svay Rieng & Ref & & \\
Age & & & \\
21-30 & 13.1 & $(7.5,18.6)$ & $<0.001$ \\
$31-40$ & 9.0 & $(2.8,15.1)$ & 0.004 \\
$41-50$ & 0.3 & $(-5.3,5.9)$ & 0.91 \\
51-60 & Ref & & \\
Qualification & & & 0.002 \\
Bachelor MW / Ns & 15.9 & $(6.0,25.8)$ & 0.015 \\
Secondary MW & 11.2 & $(2.2,20.2)$ & 0.46 \\
Primary MW & 3.4 & $(-5.7,12.5)$ & \\
Secondary / Primary Ns & Ref & & 0.47 \\
Previous training experience & & & 0.98 \\
HC-MW & 1.45 & $(-2.5,5.4)$ & \\
Partograph & 0.05 & $(-3.3,3.4)$ & \\
\hline
\end{tabular}

\section{Discussion}

This study shows that the level of knowledge necessary to monitor and evaluate the labour process is low among skilled birth attendants in the study area. Younger age and higher levels of qualification were significantly associated with higher knowledge. Those who work in Kampong Cham scored significantly higher than those in the other two regions. Previous training courses did not contribute to the current knowledge level.

The total numbers of deliveries in the study participant facilities in 2017 were 8950, 12,860, and 8509 in Phnom Penh, Kampong Cham, and Svay Rieng, respectively, which accounted for 22,47 , and $61 \%$ of all births in each region. These figures indicate that a substantial proportion of deliveries were conducted by 
Table 6 Misclassification of monitoring items during labour $[n=523]$

\begin{tabular}{|c|c|c|c|c|}
\hline \multirow[t]{2}{*}{ Category } & \multirow[t]{2}{*}{ Item } & \multicolumn{3}{|c|}{ Proportion of incorrect answer } \\
\hline & & Progress of labour & Foetal condition & Maternalcondition \\
\hline \multirow[t]{3}{*}{ Progress of labour } & Cervical dilatation & & $12.2 \%$ & $41.9 \%$ \\
\hline & Uterine contraction & & $61.4 \%$ & $26.4 \%$ \\
\hline & Foetal head descent & & $16.6 \%$ & $44.4 \%$ \\
\hline \multirow[t]{3}{*}{ Foetal condition } & Foetal heart rate & $19.5 \%$ & & $26.2 \%$ \\
\hline & Amniotic fluid & $34.6 \%$ & & $18.9 \%$ \\
\hline & Moulding of foetal head & $2.1 \%$ & & $2.1 \%$ \\
\hline \multirow[t]{4}{*}{ Maternal condition } & Pulse & $22.9 \%$ & $7.1 \%$ & \\
\hline & Blood pressure & $29.3 \%$ & $5.7 \%$ & \\
\hline & Temperature & $19.9 \%$ & $5.2 \%$ & \\
\hline & Urine volume & $21.8 \%$ & $12.2 \%$ & \\
\hline
\end{tabular}

trained health staff without a higher level of knowledge on monitoring the progress of delivery. Knowledge ensures quality of care through correct gathering and appropriate interpretation of information from a pregnant woman and foetus as well as translation of knowledge into practice. Sharing information on clinical course and its management plan facilitates practicing team medicine. Referring to the framework for quality maternal and newborn care proposed by Renfrew et al. [22], a lack of basic knowledge to monitor and evaluate the labour course hampers the 'assessment of progress of labour.' Therefore, further practices will not be realised, including 'promotion of normal processes and prevention of complications' and 'first-line management of complications.' Maintaining a sufficient level of knowledge is crucial to providing quality care, which contributes to reduce unnecessary morbidity and mortality both in mothers and babies.

This study has shown that age and qualification affected the level of knowledge. Cambodia experienced genocides during the Pol-Pot regime between 1975 and 1979 following a decade of civil conflict. Severe shortage of health care professionals and schools to provide appropriate training was a main issue in health service provision in Cambodia since the early 1980s. Oneyear training for 'primary midwife' and 'primary nurse' was created in 1989 to rapidly increase the number of health personnel, although the quality of the courses was untested and questionable [23]. A systematic review on the determinants of quality midwifery care has suggested that short-term training of nurses or midwives is far from the international standard [24]. However, the primary midwife course in Cambodia was maintained until 2015. Durations of undergraduate training are three and four years for secondary and bachelor midwives, respectively
$[25,26]$. This history and duration of education could be contributing factors for higher knowledge levels among bachelor and secondary midwives than for nurses and primary midwives.

Undergraduate training was observed to be insufficient, and the competency of health care professionals may be improved by the provision of postgraduate training. However, in this study none of the selected training courses showed significant relationship with level of knowledge. Possible factors underlying this finding are either deficiency in the providers of the training or in the participants. For the provider side, it may be because the quality of the training was poor, or little effort such as supervision was made to maintain knowledge of the participants after the training. Although it is difficult to retrospectively evaluate the quality of the previous training, we have confirmed that the selected courses contained appropriate modules for monitoring of labour and delivery. Trainers for the courses were experienced medical doctors or midwives from NMCHC; therefore, low quality of training was unlikely. Studies on training experience in neonatal resuscitation have shown that knowledge and skills deteriorate in the absence of active continuous education with mentoring $[27,28]$. Studies on contributing factors of effective learning have shown that the learning environment in the workplace and supportive supervision are key issues [29, 30]. Knowledgeable and skilled preceptors are required who can facilitate other staff members. However, in our study area the average number of birth attendants is 3.5 per facility (542 eligible persons in 157 facilities), and the number of bachelor holders is limited. Because of the shift work nature of health facilities, there would be little opportunity for birth attendants to share information on and discuss findings of labour courses with their colleagues. It 
implies that it is difficult to expect to conduct self-learning activities in each facility.

The Midwifery Coordination Alliance Team (MCAT) meeting, which was initiated in 2007 in Cambodia, is a mechanism to provide a link between midwives in health centres and hospitals as well as district staff. Its principal components are supervision and feedback for problem solving for common health centre issues, discussion on referred and complicated cases, and updating knowledge to refresh clinical skills [31-33]. Although a national plan intends to scale up the MCAT meetings to all districts [34], to date activities have been organised only in areas where external financial and technical supports are available. Of the three provinces in this study, currently only Kampong Cham has regular MCAT activity [35]. This may explain the higher knowledge level in Kampong Cham than in the other two provinces, although we have no supporting evidence since there was no comparable study using control groups.

Substantial proportions of misclassification of monitoring items during labour were found in this study: $61 \%$ answered uterine contraction as a foetal condition; $44 \%$ answered foetal head descent, and $26 \%$ answered foetal heart rate as maternal conditions; and 29\% answered blood pressure as a progress of labour. The participants might have responded without due consideration and rather with an intention of increasing their score. If this is the case, it is a sign of lack of self-confidence, which is a part of professional wisdom of midwifery [36]. These findings also imply that their manner of comprehension was not well structured. As early as the year 1882, Lusk stated that midwifery practice should be based on physiological and pathological investigations, and as a natural outcome of scientific principles [37]. Science requires classification of a phenomenon to understand its background and nature. The International Confederation of Midwives defines key competencies of midwifery as assessment and care of women during labour that facilitates physiological processes and a safe birth; and basic knowledge to fulfill the competency includes anatomy of maternal pelvis and foetus, mechanisms of labour for different foetal presentations, and physiologic onset and progression of labour [38]. Therefore, the misclassifications of monitoring items during delivery imply a lack of understanding among the study participants of physiology and anatomy in pregnancy, labour, and childbirth.

There are possible limitations in this study. First is that recall bias could influence the association between previous training experiences and the knowledge level. The study participants might not declare or forget their previous attendance in training, since two training courses (Health centre midwifery course, and Partograph, the details are shown in Annex-1) were started in the late 1990s. A second possible limitation is that we have not explored other potential factors which affect knowledge and competency of health care professionals, such as the experience of having refresher training. Further studies are required to confirm facilitating factors for maintaining appropriate knowledge in medical practices.

\section{Conclusions}

Our study found that knowledge is insufficient on management of delivery among skilled birth attendants in Cambodia. Previous experience of a single training course did not influence knowledge levels, but MCAT activities might positively contribute. Case-based learning and clinical simulations with repetitive courses for in-service training are recommended to improve knowledge and skill $[39,40]$. However, considering the potential deficit in knowledge of physiology and anatomy for midwifery care, the mere provision of clinical training will not change the situation substantially. Physiology and anatomy are foundations of clinical diagnosis, which aims to distinguish pathological states from normal conditions. Building skills and competencies for managing both normal and complicated deliveries is not realistic without a strong background in knowledge of anatomy and physiology. We speculate that the lack of knowledge in basic science abets unnecessary interventions and over-medicalisation in maternity care in Cambodia [41]. Restructuring both pre- and in-service training is required to overcome the constraint in knowledge. Training courses on delivery care should facilitate understanding the mechanism of labour progress as well as its foundation in physiology and anatomy. Studies have suggested that the introduction of conceptual models and core principles of basic science in clinical training would facilitate interplay of knowledge and experience, which enables the participants to explain 'what she is doing and why' [36, 42, 43]. Further intervention with its evaluation should be attempted to improve the knowledge of intrapartum care among birth attendants and to provide quality maternity services in Cambodia.

\section{Abbreviations}

FIGO: International Federation of Gynecology and Obstetrics; ICM: International Confederation of Midwives; ICN: International Council of Nurses; IINeoC: Project for Improving Continuum of Care with focus on Intrapartum and Neonatal Care; IPA: International Pediatric Association; JICA: Japan International Cooperation Agency; MCAT: Midwifery Coordination Alliance Team; MDGs: Millennium Development Goals; NMCHC: National Maternal and Child Health Centre; SBA: Skilled Birth Attendant; UNFPA: United Nations Population Fund; UNICEF: United Nations Children's Fund; WHO: World Health Organization. 


\section{Supplementary Information}

The online version contains supplementary material available at https://doi. org/10.1186/s12978-021-01166-z.

Additional file 1: Annex 1. Details of training in delivery care involved in this study.

\section{Acknowledgements}

The authors are indebted to Professor Vincent De Brouwere for his valuable comments on the manuscript. The authors greatly thank Professor Koum Kanal, Professor Keth Ly Sotha, Associate Professor Uong Sokhan, Associate Professor Pech Sothy, and Associate Professor Som Vanrithy from the Cambodian Society of Gynaecology and Obstetrics; and Ms Chhay Sveng Chea Ath, Ms Oung Lida, Ms Chhin Soknay, and Ms Heng Ngim from the Cambodian Midwives Association for their intensive input on developing the questionnaire. We would like also to thank Dr Thomas Templeton for English language editing.

\section{Authors' contributions}

MM and Al designed the study and wrote the manuscript; YS, YM, MM, RT, and Al created the data collection protocol; RP, BT, CN RT, YM, YS, and Al conducted the data collection; and MM performed statistical analysis. All authors read and approved the final manuscript.

\section{Funding}

This study was funded by Japan Society for the Promotion of Science (JSPS) KAKENHI Grant Number $15 \mathrm{H} 05302$ to MM; The Toyota Foundation Research Grant Program number D15-R-0447 to MM; and JICA. The funding bodies had no role in the study design; the collection, analysis, and interpretation of the data; and writing of this work.

\section{Availability of data and materials}

The datasets generated and used for this study are available from the corresponding author on reasonable request.

\section{Declarations}

\section{Ethics approval and consent to participate}

The research protocol was submitted to and approved by the National Ethics Committee for Health Research in the Ministry of Health in Cambodia (approved number 064NECHR and 139NECHR) and the Ethics Committee at Nagasaki University School of Tropical Medicine and Global Health in Japan (approved number NU-TMGH_045). The objective of this study was informed to the participants within an invitation sent to them as well as before starting the questionnaire. Written informed consent was obtained from each participant.

\section{Consent for publication}

Not applicable.

\section{Competing interests}

All the authors declare that they have no competing interests.

\section{Author details}

${ }^{1}$ Department of Global Health, Nagasaki University School of Tropical Medicine and Global Health, Sakamoto 1-12-4, Nagasaki 852-8523, Japan. ${ }^{2}$ Phnom Penh Municipal Health Department, Ministry of Health, Street 2011, Sangkat Phnom Penh Thmey, Khan Sensok, Phnom Penh, Cambodia. ${ }^{3}$ Project for Improving Continuum of Care with focus on Intrapartum and Neonatal Care in Cambodia, Japan International Cooperation Agency, Building 61-64, Preah Norodom Blvd, Phnom Penh, Cambodia. ${ }^{4}$ Kampong Cham Provincial Health Department, Ministry of Health, Preah Kosamak Nearyroth, Kampong Cham, Cambodia. ${ }^{5}$ Svay Rieng Provincial Health Department, Ministry of Health, National Road \#1, Svay Rieng, Cambodia. ${ }^{6}$ National Maternal and Child Health Center, France Street, Sangkat Srah Chork, Khan Daun Penh, Phnom Penh, Cambodia. ${ }^{7}$ Bureau of International Health Cooperation, National Center for Global Health and Medicine, Toyama 1-21-1, Shinjuku-ku, Tokyo 162-8655, Japan.
Received: 12 January 2021 Accepted: 24 May 2021

Published online: 09 June 2021

\section{References}

1. Ayres-de-Campos D, Arulkumaran S, FIGO Intrapartum Fetal Monitoring Expert Consensus Panel. FIGO consensus guidelines on intrapartum fetal monitoring: Physiology of fetal oxygenation and the main goals of intrapartum fetal monitoring. Int J Gynaecol Obstet. 2015;131(1):5-8.

2. Alliance for Maternal and Newborn Health Improvement (AMANHI) mortality study group. Population-based rates, timing, and causes of maternal deaths, stillbirths, and neonatal deaths in south Asia and sub-Saharan Africa: a multi-country prospective cohort study. Lancet Glob Health. 2018;6(12):e1297-308.

3. Kassebaum NJ, Bertozzi-Villa A, Coggeshall MS, Shackelford KA, Steiner C, Heuton KR, et al. Global, regional, and national levels and causes of maternal mortality during 1990-2013: a systematic analysis for the Global Burden of Disease Study 2013. Lancet. 2014;384(9947):980-1004.

4. World Health Organization Maternal Health and Safe Motherhood Programme. World Health Organization partograph in management of labour. Lancet. 1994;343(8910):1399-404.

5. World Health Organization. WHO recommendations: intrapartum care for a positive childbirth experience. Geneva: World Health Organization; 2018.

6. Bernitz S, Dalbye R, Zhang J, Eggebø TM, Frøslie KF, Olsen IC, et al. The frequency of intrapartum caesarean section use with the WHO partograph versus Zhang's guideline in the Labour Progression Study (LaPS): a multicentre, cluster-randomised controlled trial. Lancet. 2019;393(10169):340-8

7. Qureshi ZP, Sekadde-Kigondu C, Mutiso SM. Rapid assessment of partograph utilisation in selected maternity units in Kenya. East Afr Med J. 2010;87(6):235-41.

8. Opiah MM, Ofi AB, Essien EJ, Monjok E. Knowledge and utilization of the partograph among midwives in the Niger Delta Region of Nigeria. Afr J Reprod Health. 2012;16(1):125-32.

9. Yisma E, Dessalegn B, Astatkie A, Fesseha N. Knowledge and utilization of partograph among obstetric care givers in public health institutions of Addis Ababa Ethiopia. BMC Pregnancy Childbirth. 2013;13:17.

10. Asibong U, Okokon IB, Agan TU, Oku A, Opiah M, Essien EJ, et al. The use of the partograph in labor monitoring: a cross-sectional study among obstetric caregivers in General Hospital, Calabar, Cross River State Nigeria. Int J Womens Health. 2014;6:873-80.

11. Khan ANS, Billah SM, Mannan I, Mannan II, Begum T, Khan MA, et al. A cross-sectional study of partograph utilization as a decision making tool for referral of abnormal labour in primary health care facilities of Bangladesh. PLoS ONE. 2018;13(9):e0203617.

12. Ministry of Planning Cambodia. Annual progress report-achieving the millennium development goals report prepared on the status in 2013. Phnom Penh: Ministry of Planning; 2013.

13. National Institute of Statistics, Directorate General for Health, ICF International. Cambodia Demographic and Health Survey. USA: National Institute of Statistics, Directorate General for Health, ICF International; 2014. p. 2015

14. Ministry of Health Cambodia. Health strategic plan 2016-2020. Phnom Penh: Ministry of Health; 2016.

15. World Health Organization. Definition of skilled health personnel providing care during childbirth the 2018 joint statement by WHO, UNFPA, UNICEF, ICM, ICN, FIGO, IPA. Geneva: World Health Organization; 2018.

16. Harvey S, Blandón YC, McCaw-Binns A, Sandino I, Urbina L, Rodríguez C, et al. Are skilled birth attendants really skilled? A measurement method, some disturbing results and a potential way forward. Bull World Health Organ. 2007;85(10):783-90.

17. Liljestrand J, Moore J, Tholandi M. Active management of the third stage of labor and eclampsia management as critical components of skilled care during birth in Cambodia. Int J Gynaecol Obstet. 2010;111(2):188-9.

18. Sandin-Bojö AK, Hashimoto M, Kanal K, Sugiura Y. Intrapartum care at a tertiary hospital in Cambodia: a survey using the Bologna Score. Midwifery. 2012;28(6):e880-5. 
19. Ith P, Dawson A, Homer CS, Klinken WA. Practices of skilled birth attendants during labour, birth and the immediate postpartum period in Cambodia. Midwifery. 2013;29(4):300-7.

20. Masuda C, Ferolin SK, Masuda K, Smith C, Matsui M. Evidence-based intrapartum practice and its associated factors at a tertiary teaching hospital in the Philippines, a descriptive mixed-methods study. BMC Pregnancy Childbirth. 2020:20(1):78.

21. Ministry of Health Cambodia. Safe motherhood clinical management national protocol-health centers. Phnom Penh: Ministry of Health; 2016.

22. Renfrew MJ, McFadden A, Bastos MH, Campbell J, Channon AA, Cheung NF, et al. Midwifery and quality care: findings from a new evidence-informed framework for maternal and newborn care. Lancet. 2014;384(9948):1129-45.

23. Heng MB, Key PJ. Cambodian health in transition. BMJ. 1995;311(7002):435-7.

24. Fillby A, McConville F, Portela A. What prevents quality midwifery care? A systematic mapping of barriers in low and middle income countries from the provider perspective. PLoS ONE. 2016;11(5):e0153391.

25. Ministry of Health Cambodia. PMNCH, WHO, World Bank, AHPSR, participants in the Cambodia multistakeholder policy review Success Factors for Women's and Children's Health: Cambodia. Geneva: World Health Organization; 2014.

26. Law KM-H, Te V, Hill PS. Cambodia's health professionals and the ASEAN Mutual Recognition Arrangements: registration, education and mobility. Hum Resour Health. 2019;17(1):14.

27. Singhal N, Lockyer J, Fidler H, Keenan W, Little G, Bucher S, et al. Helping Babies Breathe: global neonatal resuscitation program development and formative educational evaluation. Resuscitation. 2012;83(1):90-6.

28. Bang A, Patel A, Bellad R, Gisore P, Goudar SS, Esamai F, et al. Helping Babies Breathe (HBB) training: What happens to knowledge and skills over time? BMC Pregnancy Childbirth. 2016;16(1):364.

29. Shankar A, Sebayang S, Guarenti L, Utomo B, Islam M, Fauveau V, et al. The village-based midwife programme in Indonesia. Lancet. 2008;371(9620):1226-9.

30. Kwast $B E$, Bentley J. Introducing confident midwives: midwifery education-action for safe motherhood. Midwifery. 1991;7(1):8-19.

31. Van Lerberghe W, Matthews Z, Achadi E, Ancona C, Campbell J, Channon $A$, et al. Country experience with strengthening of health systems and deployment of midwives in countries with high maternal mortality. Lancet. 2014;384(9949):1215-25.

32. Campbell OM, Calvert C, Testa A, Strehlow M, Benova L, Keyes E, et al. The scale, scope, coverage, and capability of childbirth care. Lancet. 2016;388(10056):2193-208.
33. University Research Co. Transitioning the Cambodian health system to self-reliance: URC's contribution 2002-2019. Chevy Chase, MD : University Research Co., LLC; 2019. https://www.urc-chs.com/sites/default/files/ URC\%20Cambodia\%20HSS\%20legacy\%20Capacity\%20v7-USAID.pdf. Accessed 4 Feb 2020.

34. Ministry of Health Cambodia. National strategy for reproductive and sexual health in Cambodia 2017-2020. Phnom Penh: Ministry of Health. 2017.

35. Liljestrand J, Sambath MR. Socio-economic improvements and health system strengthening of maternity care are contributing to maternal mortality reduction in Cambodia. Reprod Health Matters. 2012;20(39):62-72.

36. Halldorsdottir S, Karlsdottir SI. The primacy of the good midwife in midwifery services: an evolving theory of professionalism in midwifery. Scand J Caring Sci. 2011;25(4):806-17.

37. Lusk WT. The science and art of midwifery. London: HK Lewis; 1882.

38. International Confederation of Midwives. Essential Competencies for Midwifery Practice 2019 update. The Hague: International Confederation of Midwives; 2019. https://www.internationalmidwives.org/our-work/ policy-and-practice/essential-competencies-for-midwifery-practice.html. Accessed 29 Apr 2021.

39. Cooper S, Cant R, Porter J, Bogossian F, McKenna L, Brady S, Fox-Young S. Simulation based learning in midwifery education: a systematic review. Women Birth. 2012;25(2):64-78.

40. Bluestone J, Johnson P, Fullerton J, Carr C, Alderman J, BonTempo J. Effective in-service training design and delivery: evidence from an integrative literature review. Hum Resour Health. 2013;11:51.

41. Miller S, Abalos E, Chamillard M, Ciapponi A, Colaci D, Comande D, et al. Beyond too little, too late and too much, too soon: a pathway towards evidence-based, respectful maternity care worldwide. Lancet. 2016;388(10056):2176-92

42. Modell HI. How to help students understand physiology? Emphasize general models. Adv Physiol Educ. 2000;23(1):101-7.

43. Michael J, Modell H, McFarland J, Cliff W. The, "core principles" of physiology: what should students understand? Adv Physiol Educ. 2009;33(1):10-6.

\section{Publisher's Note}

Springer Nature remains neutral with regard to jurisdictional claims in published maps and institutional affiliations.
Ready to submit your research? Choose BMC and benefit from:

- fast, convenient online submission

- thorough peer review by experienced researchers in your field

- rapid publication on acceptance

- support for research data, including large and complex data types

- gold Open Access which fosters wider collaboration and increased citations

- maximum visibility for your research: over $100 \mathrm{M}$ website views per year

At BMC, research is always in progress.

Learn more biomedcentral.com/submissions 